\title{
Correlation of Lipid Parameters and Markers of Insulin Resistance: Does Smoking Make a Difference?
}

\author{
L. CIBIČKOVÁ ${ }^{1}$, D. KARÁSEK ${ }^{1}$, K. LANGOVÁ ${ }^{2}$, H. VAVERKOVÁ ${ }^{1}, J_{.}$ORSÁG $^{1}$, \\ J. LUKES $\check{S}^{3}$, D. NOVOTNÝ ${ }^{3}$
}

${ }^{1}$ Third Department of Internal Medicine - Nephrology, Rheumatology and Endocrinology, Faculty of Medicine and Dentistry, Palacky University, Olomouc, Czech Republic, ${ }^{2}$ Department of Medical Biophysics, Faculty of Medicine and Dentistry, Palacky University, Olomouc, Czech Republic, ${ }^{3}$ Department of Clinical Biochemistry, University Hospital, Olomouc, Czech Republic

Received July 10, 2014

Accepted July 23, 2014

\begin{abstract}
Summary
Insulin resistance associated with dyslipidemia enhances cardiovascular risk. Several atherogenic indexes have been suggested to give more precise information about the risk. The aim of our study was to estimate, which atherogenic index correlates better with parameters of insulin resistance. Furthermore, we compared the parameters of lipid metabolism and insulin resistance between smokers and non-smokers. In our cross-sectional study we enrolled 729 patients with dyslipidemia which were divided into two groups - non-smokers (586) and smokers (143). We measured lipid profile, parameters of insulin resistance (fasting glycemia, insulin, HOMA-IR, C-peptide, proinsulin) and calculated atherogenic indexes - atherogenic index of plasma (log (TAG/HDL-C), AIP), ApoB/ApoA1 index and nonHDL-C. AIP was found out to show stronger correlations with parameters of insulin resistance $(p<0.001$, correlation coefficients ranging between 0.457 and 0.243 ) than other indexes (ApoB/ApoA1 or nonHDL cholesterol). AIP correlated with parameters of insulin resistance both in smokers and nonsmokers, but after adjustment (for age, body mass index, waist circumference) persisting only in non-smokers. Smokers had a wider waist circumference and a proatherogenic lipid profile. Smoking increases the risk of developing metabolic syndrome. AIP can be used in daily praxis for predicting insulin resistance in patients with dyslipidemia, predominantly in non-smokers.
\end{abstract}

\section{Key words}

Insulin resistance - Metabolic syndrome • Lipid metabolism • Smoking habit $\bullet$ Atherogenic index

\section{Corresponding author}

L. Cibičková, Third Department of Internal Medicine Nephrology, Rheumatology and Endocrinology, University Hospital, I. P. Pavlova 6, 77900 Olomouc, Czech Republic. Fax: +420 588442526. E-mail: Cibickova@seznam.cz

\section{Introduction}

The incidence of metabolic syndrome is increasing worldwide. Although insulin resistance is crucial to the pathogenesis of this syndrome, the associated atherogenic lipoprotein phenotype considerably enhances the risk of cardiovascular complications. Several so called atherogenic indexes have been suggested to give more precise information about the present cardiovascular risk. These indexes are calculated from measured values of plasma lipids. One of the recommended indexes is the atherogenic index of plasma (AIP). AIP is calculated as a log (TAG/HDL-C) with triacylglycerols (TAG) and high density lipoprotein cholesterol (HDL-C) expressed in molar concentrations and was proposed by Dobiasova et al. (2001) and Frohlich et al. (2003). The association of TAG and HDL-C in this ratio reflects the balance between risk and protective lipoprotein forces. Both TAG and HDL-C are widely measured and available. Low HDL-C and high TAG concentrations induce both an increase in small HDL particle and an increase in small, dense low density lipoprotein cholesterol (LDL-C) particles (Dobiasova 2004). AIP has been found to be an important tool for

PHYSIOLOGICAL RESEARCH • ISSN 0862-8408 (print) • ISSN 1802-9973 (online)

(C) 2014 Institute of Physiology v.v.i., Academy of Sciences of the Czech Republic, Prague, Czech Republic

Fax+420 241062 164, e-mail: physres@biomed.cas.cz, www.biomed.cas.cz/physiolres 
analyzing the results of clinical trials, assessing not only cardiovascular risk, but also changes in the lipoprotein profile during therapy of diabetes (Dobiasova 2004, Tan et al. 2004, Essiarab et al. 2014, Zhu et al. 2014).

Comparing LDL-C and non HDL-C, non HDL-C has been shown to be a better marker of risk both in primary and secondary prevention studies (Virani 2011). In an analysis of data combined form 68 studies, non HDL-C was the best predictor among cholesterol measures, both for cardiovascular events and for strokes (Di Angelantonio et al. 2009). Another effective predictor of coronary heart disease risk is apolipoprotein $\mathrm{B} /$ apolipoprotein $\mathrm{A} 1$ index (ApoB/ApoA1), especially in overweight and obesity (Lu et al. 2011). It is associated with early atherosclerosis (Panayiotou et al. 2008) and estimates the balance between plasma proatherogenic and antiatherogenic lipoproteins (Walldius et al. 2004).

In our cross sectional study, we compared AIP with other above mentioned atherogenic indexes (ApoB/ApoA1 and non HDL-C) in order to find out, which atherogenic index correlates better with parameters of insulin resistance. Furthermore, we split up our study group into smokers and non-smokers, and compared the parameters of lipid metabolism and insulin resistance between these two groups.

The reason for this sorting was the fact, that smoking may influence parameters of both lipid and glucose metabolism by reducing insulin sensitivity and inducing insulin resistance (e.g. Targher et al. 1997, LathiKoski et al. 2002). Smoking contributes to advancement of metabolic syndrome in several ways: it stimulates sympatic nervous activity and enhances energetic expenditure. $\mathrm{Via}$ increasing of lipomobilisation, it can increase the supply of fatty acids to liver, skeletal muscles and stimulate growth of visceral fatty tissue (Kim et al. 2012). This situation is worsened by negative influence of smoking on 11 betahydroxysteroid dehydrogenase (Al Bakir et al. 2008) that enables inactivation of cortisol on cortisone. This leads to hypercortisolism which accentuates negative impact of increasing visceral fat.

\section{Methods}

729 patients (350 men and 379 women) of the Lipid Center at the University Hospital Olomouc who came for their first visit because of hyperlipidemia (total cholesterol $\geq 5 \mathrm{mmol} / \mathrm{l}$ and/or TAG $\geq 1.7 \mathrm{mmol} / \mathrm{l}$ ) between January 2005 and January 2013 were included in the study. Detailed medical history was obtained and physical examination performed. All subjects were tested for secondary hyperlipidemia, particularly diabetes mellitus, hypothyroidism, hepatic or renal failure. Exclusion criteria were as follows: lipid lowering therapy in previous 6 weeks, the presence of diabetes mellitus or other secondary hyperlipidemias, acute infection or trauma, acute cardiovascular event in the last 3 months, and heart failure NYHA III and IV. Patients were asked about their smoking secession and divided into smokers and nonsmokers, whereas an ex-smoker was recorded as a nonsmoker when he quit at a young age or had not smoked for a substantial time period (Marston et al. 2014).

The study was reviewed and approved by the institutional Ethics Committee of the Medical Faculty and University Hospital Olomouc and the informed consent was obtained from all subjects.

Venous blood samples were drawn after a $12 \mathrm{~h}$ of overnight fast. Total cholesterol (TC) and TAG concentrations were measured by standard enzymatic methods (CHOD-PAP and GPO-PAP; Roche Diagnostics, Basel, Switzerland). HDL-C was measured by a direct method (both from Roche Diagnostics, Basel, Switzerland). All assays were performed in a COBAS c8000 biochemical analyzer from Roche. LDL-C levels were calculated according to Friewald formula. AIP was calculated as a $\log$ (TAG/HDL-C) (Frohlich et al. 2003) and non HDL-C as TC-HDL-C. Concentration of ApoB and apolipoprotein A1 (ApoA1) was determined immunoturbidimetrically on Modular SWA analyzer (TinaQuant Apo A1, TinaQuant Apo B kits, all Roche, Basel, Switzerland). Glycemia was determined using the enzyme based Glucose GOD-PAP kit. Insulin was determined using Insuline kit (Immunotech, Marseille, France) with specific antibodies by IRMA method. The result obtained were then used for calculation of HOMA-IR (homeostatis model assessment: fasting glycemia*fasting insulin/22.5) (Matthews et al. 1985). C-peptide and proinsulin were determined using the commercially available kit - C-peptide (Immunotech, Marseille, France), Proinsulin (DRG Instruments GmbH, Marburg, Germany) using specific anti-bodies by IRMA method (for C-peptide) and RIA method (for proinsulin). Parameters with normal distribution (normality tested with Kolmogorov-Smirnov test) were compared with Student's t-test (patients' age) and expressed as mean $\pm \mathrm{SD}$. Parameters with skewed distribution (BMI, waist circumference, total cholesterol, TAG, HDL-C, LDL-C, ApoB, glycemia, insulin, HOMA-IR, C-peptide, proinsulin) were analyzed with Mann-Whitney U-tests and 
expressed as median $\left(1^{\text {st }}-3^{\text {rd }}\right.$ quartile). Spearman's correlation and partial correlation coefficient was used in order to determine the association among parameters of insulin resistance and lipid metabolism and also for testing difference between correlation coefficients. Stepwise Forward LR model was used for logistic regression.

\section{Results}

We divided our study group into 586 nonsmokers and 143 smokers. The mean age of smokers $(44.3 \pm 13.5$ years $)$ and non-smokers $(45.6 \pm 15.5$ years $)$ did not significantly differ between groups. Both groups did not differ in body mass index (BMI), but smokers had a wider waist circumference, showing higher amount of visceral fat in smokers. Overall smokers had a proatherogenic lipid profile (higher TC, TAG, AIP, nonHDL-C, ApoB and lower HDL-C, ApoA1) and also statistically significant worse parameters of insulin resistance (fasting glycemia, insulin, HOMA-IR, C-peptide, proinsulin). Data are presented in Table 1.

AIP correlated better with all parameters of insulin resistance in comparison with $\mathrm{ApoB} / \mathrm{ApoA} 1$ index and nonHDL-C both in the whole group and also both in smokers and non-smokers, but after adjustment (for age, BMI, waist circumference, sex) persisting only in nonsmokers, as shown in Table 2. Testing difference between atherogenic indexes (AIP versus non HDL and AIP versus ApoB/ApoA1 showed significant differences between them $(\mathrm{p}<0.003)$.

According to logistic regression for predicting higher levels of glucose, age $(\mathrm{OR}=1.40, \mathrm{p}<0.0005,95 \%$ confidence interval for OR 1.17-1.66), level of TAG $(\mathrm{OR}=1.13, \mathrm{p}<0.005,95 \%$ confidence interval for $\mathrm{OR}$ 1.04-1.23) and waist circumference $(\mathrm{OR}=1.67, \mathrm{p}<0.0001$, $95 \%$ confidence interval for OR 1.38-2.02) have been shown as the most relevant predictors, where OR is counted for change in age of 10 years, in TAG of $1 \mathrm{mmol} / 1$ and $10 \mathrm{~cm}$ in waist circumference. TAG $(\mathrm{OR}=1.32, \mathrm{p}<0.005,95 \%$ confidence interval for $\mathrm{OR}$ 1.09-1.61) and waist circumference $(\mathrm{OR}=1.52, \mathrm{p}<0.0001$, $95 \%$ confidence interval for OR 1.26-1.82) are the most relevant predictors of insulin level. Waist circumference showed in both cases most significant correlations.

Table 1. Parameters of lipid metabolism and insulin resistance in smokers and non-smokers.

\begin{tabular}{llll}
\hline & Whole group & Non-smokers & Smokers \\
\hline BMI $\left(\mathrm{kg} / \mathrm{m}^{2}\right)$ & $25.5(23-28.6)$ & $25.4(23-28.3)$ & $26.3(23.1-29.4)$ \\
Age $($ years $)$ & $45.3 \pm 15.2$ & $45.6 \pm 15.5$ & $44.3 \pm 13.5$ \\
Sex $($ female/male $)$ & 379 female & 319 female & 60 female \\
& 350 male & 257 male & 83 male \\
Waist circumference $(\mathrm{cm})$ & $87(77-97)$ & $86(76-95)$ & $90(79-99)+$ \\
Total cholesterol $(\mathrm{mmol} / \mathrm{l})$ & $6.13(5.16-7.34)$ & $6.08(5.14-7.21)$ & $6.48(5.43-7.87) *$ \\
Triacylglycerols $(\mathrm{mmol} / \mathrm{l})$ & $1.68(1.12-2.65)$ & $1.59(1.09-2.34)$ & $2.51(1.39-4.21) * * *$ \\
AIP & $0.06(-0.15-0.33)$ & $0.04(-0.17-0.27)$ & $0.29(-0.05-0.67) * * *$ \\
non HDL-C $(\mathrm{mmol} / \mathrm{l})$ & $4.60(3.69-5.82)$ & $4.56(3.63-5.67)$ & $4.99(4.06-6.41) * *$ \\
LDL-C $(\mathrm{mmol} / \mathrm{l})$ & $3.67(2.79-4.63)$ & $3.69(2.88-4.58)$ & $3.65(2.64-5.02)$ \\
ApoB $(\mathrm{g} / \mathrm{l})$ & $1.13(0.93-1.37)$ & $1.12(0.91-1.35)$ & $1.18(1.00-1.45) *$ \\
HDL-C $(\mathrm{mmol} / \mathrm{l})$ & $1.41(1.15-1.73)$ & $1.44(1.19-1.75)$ & $1.30(0.99-1.67) * *$ \\
ApoAl $(\mathrm{g} / \mathrm{l})$ & $1.52(1.34-1.76)$ & $1.53(1.35-1.77)$ & $1.49(1.24-1.70)+$ \\
ApoB/ApoAl & $0.74(0.56-0.93)$ & $0.72(0.55-0.91)$ & $0.83(0.64-1.02) * * *$ \\
Fasting glycemia $(\mathrm{mmol} / \mathrm{l})$ & $5.00(4.60-5.43)$ & $4.94(4.60-5.40)$ & $5.10(4.70-5.60) *$ \\
Insulin $(\mathrm{mIU} / \mathrm{l})$ & $7.60(5.30-10.90)$ & $7.40(5.30-10.6)$ & $8.60(5.00-13.43)+$ \\
HOMA-IR & $1.68(1.11-2.58)$ & $1.63(1.11-2.40)$ & $1.99(1.11-3.08)+$ \\
C-peptide $(\mathrm{mg} / \mathrm{l})$ & $2.18(1.53-2.98)$ & $2.11(1.50-2.88)$ & $2.54(1.76-3.53) * *$ \\
Proinsulin $(\mathrm{mIU} / \mathrm{l})$ & $11.00(8.20-15.70)$ & $10.8(8.0-15.4)$ & $11.9(8.8-18.8) *$ \\
\hline
\end{tabular}

Data are expressed as median $\left(1^{\text {st }}-3^{\text {rd }}\right.$ quartile) with a $\mathrm{p}$-value giving the statistical significance of the difference between smokers and non-smokers. $+\mathrm{p}<0.05, * \mathrm{p}<0.01, * * \mathrm{p}<0.001, * * * \mathrm{p}<0.0001$, except of data about age that are expressed as mean \pm standard deviation. 
Table 2. Spearman correlation coefficient of AIP, ApoB/ApoA1 and nonHDL-C with parameters of insulin resistance in smokers and non-smokers.

\begin{tabular}{|c|c|c|c|c|c|}
\hline & Fasting glucose & Insulin & HOMA-IR & C-peptide & Proinsulin \\
\hline \multicolumn{6}{|l|}{ AIP } \\
\hline in smokers & $0.313 * *$ & $0.521 * * *$ & $0.540 * * *$ & $0.510 * * *$ & $0.509 * * *$ \\
\hline (after adjustment) & 0.091 & 0.121 & 0.137 & 0.110 & 0.073 \\
\hline in non-smokers & $0.240 * * *$ & $0.353 * * *$ & $0.369 * * *$ & $0.447 * * *$ & $0.332 * * *$ \\
\hline (after adjustment) & $0.092+$ & $0.129 *$ & $0.109 *$ & $0.220 * * *$ & $0.122 *$ \\
\hline in men & $0.211 * *$ & $0.433 * * *$ & $0.434 * * *$ & $0.489 * * *$ & $0.429 * * *$ \\
\hline in women & $0.233 * * *$ & $0.355 * * *$ & $0.381 * * *$ & $0.455 * * *$ & $0.254 * * *$ \\
\hline \multicolumn{6}{|l|}{ АроВ/Аро $A 1$} \\
\hline in smokers & 0.101 & $0.238 *$ & $0.255 *$ & $0.389 * * *$ & $0.263 *$ \\
\hline (after adjustment) & 0.050 & 0.001 & 0.036 & $0.252+$ & 0.100 \\
\hline in non-smokers & $0.145 *$ & $0.214 * * *$ & $0.222 * * *$ & $0.220 * *$ & $0.186 * * *$ \\
\hline (after adjustment) & 0.010 & 0.036 & 0.009 & 0.019 & 0.029 \\
\hline in men & 0.038 & $0.299 * * *$ & $0.278 * * *$ & $0.272 * * *$ & $0.258 * * *$ \\
\hline in women & $0.163 *$ & $0.120+$ & $0.147 *$ & $0.225 * * *$ & 0.092 \\
\hline \multicolumn{6}{|l|}{ nonHDL-C } \\
\hline in smokers & $0.310 * *$ & 0.093 & 0.158 & $0.234 *$ & 0.172 \\
\hline (after adjustment) & $0.491 * * *$ & 0.112 & 0.137 & 0.022 & 0.095 \\
\hline in non-smokers & $0.105+$ & $0.148 *$ & $0.149 *$ & $0.166 * *$ & $0.120 *$ \\
\hline (after adjustment) & 0.031 & 0.016 & 0.023 & 0.008 & 0.016 \\
\hline in men & $0.143+$ & $0.142+$ & $0.151 *$ & $0.180 *$ & $0.125+$ \\
\hline in women & $0.154 *$ & $0.126+$ & $0.143 *$ & $0.187 * *$ & 0.109 \\
\hline
\end{tabular}

$+p<0.05, * p<0.01, * * p<0.001, * * * p<0.0001$

\section{Discussion}

In our study, we have tried to find out which of the used atherogenic indexes would correlate better with parameters of insulin resistance. AIP was found out to show stronger correlations than other indexes (ApoB/ApoA1 or non HDL cholesterol). The reason for this is probably the known correlation between glucose and triglyceride plasma levels, described already in 1983 by Pfeifer et al. AIP is the only index taking into account not only HDL cholesterol levels but also plasma TAG, that play the role of a regulator of lipoprotein interactions and not the role of an independent risk marker. This claim is supported by evidence that an increased plasma concentration of TAG is associated with an increased incidence of coronary artery disease. As mentioned above, high TAG and low HDL-C concentrations induce both an increase in small HDL particle and an increase in small, dense LDL particles (Dobiasova 2004), which is especially important in patients with insulin resistance.
Thus, AIP has been found to be suitable and statistically reliable also in diabetics (Tan et al. 2004). We have shown that AIP correlates well with parameters of insulin resistance.

Nevertheless, after adjustment on age, BMI and waist circumference, power of these correlations was attenuated both in smokers and non-smokers and lost its statistical significance in smokers, suggesting higher importance of these factors. Both groups did significantly differ neither in age nor in BMI, but in waist circumference. We deduce that waist circumference drives this difference - being larger in smokers. Larger waist circumference reflects insulin resistance that is pronounced in smokers, and adjustment for waist circumference in them probably caused the loss of statistically significant correlation of AIP with parameters of insulin resistance. Another reason for loosing this correlation may be much smaller number of patients who smoked.

Larger waist circumference in smokers reflects 
accumulation of visceral tissue in these patients. In concordance with our finding, it has been published that smokers have a higher tendency for accumulating visceral tissue (Nakanishi et al. 2014). In contrast with this finding, several studies report that smokers have a lower BMI than non-smokers (e.g. Pednekar et al. 2006). In our study, we have not found any statistically significant difference in BMI of smokers and non-smokers (although there was a tendency for higher BMI in smokers in contrast to non-smokers). Nevertheless waist circumference correlates better with the amount of visceral tissue than BMI and is nowadays accepted as a part of metabolic syndrome assessment.

Smoking is accepted as a major risk factor not only for metabolic but also for cardiovascular disease (Villablanca et al. 2000, Marshall et al. 2001, WHO 2002). It enhances cardiovascular risk factors such as elevated plasma triglycerides, decreases high-density lipoprotein cholesterol (HDL-C) and causes hyperglycemia (Criqui et al. 1980, Steiner et al. 1987, Tsiara et al. 2003). Our study confirms this wellestablished risk profile of smoking subjects - we showed that currently smoking patients developed proatherogenic changes in their lipidograme. Smoking also reduced insulin sensitivity, which has already been shown by some other authors (e.g. Targher et al. 1997, Lathi-Koski et al. 2002, Li et al. 2012). Li et al. (2012) have found difference between the smoking and non-smoking participants in insulin resistance, but this difference was not significant after adjustment for BMI. Fasting serum insulin levels gradually increased with increasing BMI. We also have found waist circumference as the major and most significant predictor of high insulin and glucose levels. These relations between insulin resistance and obesity are currently intensively studied (Novotny et al. 2014).

We are aware that our study has several limitations. We have not used equally large gender and age matched groups for comparing group of smokers and non-smokers. In order to determine insulin resistance we have used HOMA-IR but not golden standard euglycemic hyperinsulinemic clamp. On the other hand HOMA-IR is suitable for daily praxis because it does not involve intravenous infusion, only simple calculation. HOMA-IR model has proved to be a robust clinical and epidemiological tool for the assessment of insulin resistance (Singh et al. 2010) and it is more frequently used than another index - so called QUICKI (Quantitative insulin sensitivity check index) (Katz et al. 2000).

We conclude that AIP can be used in daily praxis in patients with dyslipidemia, predominantly in non-smokers, as a predictor of insulin resistance. It is a simple and easily accessible laboratory parameter that correlates with markers of insulin resistance and does not need assessing of insulin level. We also showed, that smoking enhances proatherogenic lipid profile, accumulation of visceral tissue and insulin resistance and its repression is a desirable public health goal.

\section{Conflict of Interest}

There is no conflict of interest.

\section{Acknowledgements}

Supported by grant Nr. LF-2014-011.

\begin{abstract}
Abbreviations
AIP, atherogenic index of plasma; TAG, triacylglycerols; HDL-C, high density lipoprotein cholesterol; TC, total cholesterol; LDL-C, low density lipoprotein cholesterol; non HDL-C, TC-HDL-C; ApoB, apolipoprotein B; ApoA1, apolipoprotein A1; HOMA-IR, homeostatis model assessment - insulin resistance; BMI, body mass index; OR, odds ratio.
\end{abstract}

\section{References}

AL BAKIR M, BUTT AN, SWAMINATHAN R: Circulating 11beta-hydroxysteroid dehydrogenase type 1 mRNA and cardiovascular risk factors. Ann NY Acad Sci 1137: 283-289, 2008.

CRIQUI MH, WALLACE RB, HEISS G, MISHKEL M, SCHONFELD G, JONES GT: Cigarette smoking and plasma high-density lipoprotein cholesterol. The Lipid Research Clinics Program Prevalence Study. Circulation 62: 70-76, 1980.

Di ANGELANTONIO E, SARWAR N, PERRY P, KAPTOGE S, RAY KK, THOMPSON A, WOOD AM, LEWINGTON S, SATTAR N, PACKARD CJ, COLLINS R, THOMPSON SG, DANESH J: Major lipids, apolipoproteins, and risk of vascular disease. JAMA 302: 1993-2000, 2009. 
DOBIASOVA M: Atherogenic index of plasma (log (TAG/HDL-cholesterol): theoretical and practical implications. Editorial. Clin Chem 50: 1113-1115, 2004.

DOBIASOVA M, FROHLICH J: The plasma parameter log (TG/HDL-C as an atherogenic index: correlation with lipoprotein particle size and esterification rate in apoB-lipoprotein-depleted plasma $\left(\mathrm{FER}_{\mathrm{HDL}}\right)$. Clin Biochem 34: 583-588, 2001.

ESSIARAB F, TAKI H, LEBRAZI H, SABRI M, SAÏLE R: Usefulness of lipid ratios and atherogenic index of plasma in obese Moroccan women with or without metabolic syndrome. Ethn Dis 24: 207-212, 2014.

FROHLICH J, DOBIASOVA M: Fractional esterification rate of cholesterol and ratio of triglycerides to HDLcholesterol are powerful predictors of positive findings on coronary angiography. Clin Chem 49: 1873-1880, 2003.

KATZ A, NAMBI SS, MATHER K, BARON AD, FOLLMANN DA, SULLIVAN G, QUON MJ: Quantitative insulin sensitivity check index: a simple, accurate method for assessing insulin sensitivity in humans. $J$ Clin Endocrinol Metab. 85: 2402-1240, 2000.

KIM JH, SHIM KW, YOON YS, LEE SY, KIM SS, OH SW: Cigarette smoking increases abdominal and visceral obesity but not overall fatness: an observational study. PLoS One 7: e45815, 2012.

LAHTI-KOSKI M, PIETINEN P, HELIÖVAARA M, VARTIAINEN E: Associations of body mass index and obesity with physical activity, food choices, alcohol intake, and smoking in the 1982-1997 FINRISK Studies. $\mathrm{Am}$ J Clin Nutr 75: 809-817, 2002.

LI S, HUANG S, MO ZN, GAO Y, YANG XB, CHEN XJ, ZHAO JM, QIN X: Generating a reference interval for fasting serum insulin in healthy nondiabetic adult Chinese men. Singapore Med J 53: 821-825, 2012.

LU M, LU Q, ZHANG Y, TIAN G: ApoB/apoA1 is an effective predictor of coronary heart disease risk in overweight and obesity. J Biomed Res 25: 266-273, 2011.

MARSHALL RJ, STEVENSON MM: Smoking and cardiovascular disease. West Virginia Med J 97: 1720, 2001.

MARSTON L, CARPENTER JR, WALTERS KR, MORRIS RW, NAZARETH I, WHITE IR, PETERSEN I: Smoker, ex-smoker or non-smoker? The validity of routinely recorded smoking status in UZ primary care: a crosssectional study. BMJ Open 4: e004958, 2014.

MATTHEWS DR, HOSKER JP, RUDENSKI AS, NAYLOR BA, TREACHER DF, TURNER RC: Homeostasis model assessment: insulin resistance and beta-cell function from fasting plasma glucose and insulin concentrations in man. Diabetologia 28: 412-419, 1985.

NAKANISHI K, NISHIDA M, OHAMA T, MORIYAMA T, YAMAUCHI-TAKIHARA K: Smoking associates with visceral fat accumulation especially in women. Circ $J$ 78: 1259-1263, 2014.

NOVOTNY D, VAVERKOVA H, KARASEK D, LUKES J, SLAVIK L, MALINA P, ORSAG J: Evaluation of total adiponectin, adipocyte fatty acid binding protein and fibroblast growth factor 21 levels in individuals with metabolic syndrome. Physiol Res 63: 219-228, 2014.

PANAYIOTOU A, GRIFFIN M, GEORGIOU N, BOND D, TYLLIS T, TZIAKOURI-SHIAKALLI C, FESSAS C, NICOLAIDES A: ApoB/apoA1 ratio and subclinical atherosclerosis. Int Angiol 27: 74-80, 2008.

PEDNEKAR M, GUPTA P, SHUKLA H, HERBERT J: Association between tobacco use and body mass index in urban Indian population: implications for public health in India. BMC Public Health 6: 70, 2006.

PFEIFER MA, BRUNZELL JD, BEST JD, JUDZEWITSCH RG, HALTER JB, PORTE D JR: The response of plasma triglyceride, cholesterol and lipoprotein lipase to treatment in non-insulin-dependent diabetic subjects without familial hypertriglyceridemia. Diabetes 32: 525-531, 1983.

SINGH B, SAXENA A: Surrogate markers of insulin resistance: A review. World J Diabetes 15: 36-47, 2010.

STEINER G, SCHWARTZ L, SHUMAK S, POAPST M: The association of increased levels of intermediate-density lipoproteins with smoking and with coronary artery disease. Circulation 75: 124-130, 1987.

TAN MH, JOHNS D, GLAZER NB: Pioglitazone reduces atherogenic index of plasma in patients with type 2 diabetes. Clin Chem 50: 1184-1188, 2004.

TARGHER G, ALBERICHE M, ZENERE MB, BONADONNA RC, MUGGEO M, BONORA E: Cigarette smoking and insulin resistance in patients with noninsulin-dependent diabetes mellitus. J Clin Endocrinol Metab 82: 3619-3624, 1997. 
TSIARA S, ELLISAF M, MIKHAILIDS DP: Influence of smoking on predictors of vascular disease. Angiology 54: 507-530, 2003.

VILlablanCA A, MCDONALD J, RUTLEDGE J: Smoking and cardiovascular disease. Clinics Chest Med 21: 159-172, 2000.

VIRANI SS: Non-HDL cholesterol as a metric of good quality of care. Tex Heart Inst J 38: 160-162, 2011.

WALLDIUS G, JUNGNER I, AASTVEIT AH, HOLME I, FURBERG CD, SNIDERMAN AD: The apoB/apoA-I ratio is better than the cholesterol ratios to estimate the balance between plasma proatherogenic and antiatherogenic lipoproteins and to predict coronary risk. Clin Chem Lab Med 42: 1355-1363, 2004.

WORLD HEALTH ORGANIZATION: Tobacco Smoke and Involuntary Smoking. Geneva, WHO, 2002.

ZHU XW, DENG FY, LEI SF: Meta-analysis of Atherogenic Index of Plasma and other lipid parameters in relation to risk of type 2 diabetes mellitus. Prim Care Diabetes, in press 2014. 